$1.8 \mathrm{pg} / \mathrm{mL}$ ) levels than patients with rheumatoid arthritis (RA) $(1.8 \pm 1.01 \mathrm{pg} / \mathrm{mL}$ and $3.2 \pm 1.6 \mathrm{pg} / \mathrm{mL}$, respectively, $p<0.001$ for both) or HCs $(2.2 \pm 1.64 \mathrm{pg} / \mathrm{mL}$ and $2.2 \pm 1.7$ $\mathrm{pg} / \mathrm{mL}$, respectively, $p<0.001$ for both). The levels of salivary IGHG3 correlated with erythrocyte sedimentation rate $(r$ $=0.26, p=0.01)$, anti-dsDNA antibody levels $(\mathrm{r}=0.25, p$ $=0.01)$, and nephritis $(\mathrm{r}=0.28, \mathrm{p}=0.01)$.

Conclusions The levels of salivary IGHG3 and lactoferrin were higher in patients with SLE. In addition, salivary IGHG3 levels correlated with SLE disease activity markers.

Acknowledgements This research was supported by a grant from the Korea Health Technology R\&D Project through the Korea Health Industry Development Institute (KHIDI), funded by the Ministry of Health \& Welfare, Republic of Korea (HI16C0992).

\section{P41 PREDICTING AUTOIMMUNE CONNECTIVE TISSUE DISEASES: THREE YEAR FOLLOW UP OF AN AT RISK COHORT IDENTIFIES LATE PROGRESSION AND BIOMARKERS TO PREDICT NEED FOR THERAPY}

${ }^{1}$ Sabih-Ul Hassan, ${ }^{1}$ Katie Dutton, ${ }^{1}$ Zoe Wigston, ${ }^{2}$ Ade Alase, ${ }^{1}$ Md Yuzaiful Md Yusof, ${ }^{1}$ Edward M Vital. 'NIHR Leeds Biomedical Research Centre, Leeds Teaching Hospitals NHS Trust, Leeds; ${ }^{2}$ Leeds Institute of Rheumatic and Musculoskeletal Medicine, University of Leeds, Leeds, UK

\subsection{6/lupus-2020-eurolupus.89}

Background Autoimmune connective tissue diseases (AI-CTDs: SLE, pSS, IIM, Scleroderma, MCTD) are preceded by asymptomatic ANA positivity. We previously recruited a cohort of new ANA-positive referrals without AI-CTD. 17\% met criteria by AI-CTD at 12 months, and this was predicted an IFN Score. This study includes 3 year follow up of that cohort with more detailed analysis of the 'non-progressor' group and predictors.

Methods Patients were recruited if they had: (i) ANA; (ii) did not meet criteria for AI-CTD; (iii) symptoms less than 12 months. Diagnostic criteria for AI-CTDs and therapies were assessed at baseline then 12-monthly for 3 years. We categorised progression as:

1. Absolute-non-progressors (no clinical criteria at all time points: $0-36$ months)

2. Undifferentiated-CTD ( $\geq 1$ clinical criterion at baseline and/or at follow up but not meeting criteria)

3. Year-1-progressor (meeting criteria for AI-CTD within 12 months)

4. Late-progressor (meeting criteria for AI-CTD later than 12 months)

Interferon Score A and Interferon Score B were measured at baseline using taqman as previously described. ${ }^{1}$

Results 3-year follow up was available in 146/150. Proportions in the above categories were: Absolute-non-progressors: 33/146 (23\%); Undifferentiated-CTD: 86/146 (59\%); Year-1-progressors: 21/146 (14\%); Late-progressors: 5/146 $(3 \%)$. No patient progressed or required immunosuppression after 2 years. $6 / 86$ patients with Undifferentiated-CTD received an immunosuppressant. The present work therefore defines a larger group of 32/146 (22\%) with clinically significant disease including 21 Year-1-progressors, 5 late-progressors, and 6 undifferentiated-CTD who needed an immunosuppressant.
Clinical features had limited utility in predicting these outcomes. Of 31 patients with no clinical criteria at baseline, 1 progressed to meet criteria within 1 year, 2 progressed at 1-2 years, 3 were prescribed hydroxychloroquine and 1 was prescribed an immunosuppressant. The 108 patients with at least 1 criterion at baseline had the highest risk: 20 progressed to meet criteria within 1 year, 2 progressed at 1-2 years, the others all had U-CTD. 35 were prescribed hydroxychloroquine and 13 were prescribed an immunosuppressant. There was also no association between ENA, C3 or C4 and clinical outcome.

The association between interferon scores and progression was stronger when comparing Year 1 progressors with absolute non-progressors $(p=0.007)$. Late progression was not predicted by baseline IFN Scores. However, within U-CTD, patients who required an immunosuppressant had higher expression of IFN Score A $(p=0.011)$ and IFN Score B $(\mathrm{p}<0.001)$ than those who did not.

Conclusions Among ANA-positive referrals, no clinical feature or routine laboratory test could rule out development of clinically significant disease, but IFN Scores had a unique value in predicting these outcomes. At-Risk individuals who ultimately developed clinically significant disease are therefore immunologically but not clinically distinctive. Future work will incorporate biomarkers into clinically applicable risk models to allow earlier exclusion of AI-CTD or trials of preventative treatment.

Acknowledgements This project was funded by a grant from the National Institute for Health Research.

\section{P42 DISEASE ACTIVITY, IMPAIRED IRON TRANSPORT AND FAILED SEQUESTRATION: A NOVEL MECHANISM FOR ANAEMIA IN SYSTEMIC LUPUS ERYTHEMATOSUS}

Chris Wincup, Thomas McDonnell, George Robinson, Filipa Farinha, Anna Radziszewska David Isenberg, Anisur Rahman. Dept. of Rheumatology, University College London, London, UK

\subsection{6/lupus-2020-eurolupus.90}

Background Haematological manifestations are a frequent feature of systemic lupus erythematosus (SLE) yet the role of abnormal iron metabolism is poorly understood. In this study, we investigated the role of key regulators of iron metabolism including ferritin (an iron carrier protein), transferrin (which facilitate iron transport to cell surface receptors), hepcidin (which prevents iron release from stores under the influence of IL6 and IL1 $\beta$ ) and lipocalin-2 (LCN2, which is released by innate immune system activation and induces iron sequestration).

Methods Serum samples were collected from SLE patients without a history of haemolytic anaemia attending University College London Hospital, UK $(n=39)$. Clinical parameters including Haemoglobin (Hb), dsDNA, complement C3 and SLEDAI-2K were recorded. Levels of IL1 $\beta$, IL6, hepcidin, ferritin, LCN2 and transferrin in addition to other iron regulators including erythropoietin (EPO), soluble transferrin receptor (sTfR), haptoglobin $(\mathrm{Hp})$ and NRAMP2, were measured by ELISA. Following normalisation of data, hierarchical correlate cluster was performed to produce a heatmap using $\mathrm{MeV}$ Software.

Results The results demonstrate a surprising negative correlation between LCN2 and SLEDAI-2K $(\mathrm{P}<0.001, \quad \mathrm{r}=-0.40)$, which suggests iron is not being appropriately sequestered in 